EPJ Web of Conferences 23, 00009 (2012)

DOI: $10.1051 /$ epjconf/20122300009

(C) Owned by the authors, published by EDP Sciences, 2012

\title{
Observation of Anomalous Properties associated with the Low Temperature Structural Distortion in $\beta$-FeSe and Related Superconductors ${ }^{a}$
}

\author{
M. K. Wu \\ Institute of Physics, Academia Sinica, Nankang, Taipei, Taiwan
}

\begin{abstract}
The discovery of Superconductivity in the tetragonal phase FeSe provides a unique platform for the detailed investigation of the correlation between the physical properties and crystal structure to better understand the possible origin of superconductivity in the new iron-based superconductors. We have carried out a series of properties characterizations by measuring magnetic susceptibility, Raman, NMR and femtosecond spectroscopy on single crystals and epitaxial thin films of the FeSe and Te-doped Fe(SeTe) samples. Our results show clearly the presence of anomalies in all the characterized properties at the temperature where a structural distortion from tetragonal to orthorhombic (or monoclinic) appears for all superconducting samples, but not in the non-superconducting ones. This structural distortion was observed not accompanied by a magnetic ordering as commonly occurs in the parent compounds of FeAs-based superconductors. All the observations suggest that the low temperature structural distortion is essential for the occurrence of superconductivity in the FeSe and related compounds. Details of the experimental results will be presented and discussed.
\end{abstract}

a The presentation slides are available at the website
http://www.its.caltech.edu/ yehgroup/documents/ITAP2011_Conference_WuMK.pdf

This is an Open Access article distributed under the terms of the Creative Commons Attribution-Noncommercial License 3.0, which permits unrestricted use, distribution, and reproduction in any noncommercial medium, provided the original work is properly cited. 